Rhode Island College

Digital Commons @ RIC

$5-13-2021$

\title{
Identifying Nurses' Perceived Barriers to Mobilizing Patients on a Medical-Surgical Unit: A Quality Improvement Project
}

Jil Lukin

Follow this and additional works at: https://digitalcommons.ric.edu/etd

Part of the Nursing Commons

\section{Recommended Citation}

Lukin, Jil, "Identifying Nurses' Perceived Barriers to Mobilizing Patients on a Medical-Surgical Unit: A Quality Improvement Project" (2021). Master's Theses, Dissertations, Graduate Research and Major Papers Overview. 383.

https://digitalcommons.ric.edu/etd/383

This Major Paper is brought to you for free and open access by the Master's Theses, Dissertations, Graduate Research and Major Papers at Digital Commons @ RIC. It has been accepted for inclusion in Master's Theses, Dissertations, Graduate Research and Major Papers Overview by an authorized administrator of Digital Commons @ RIC. For more information, please contact digitalcommons@ric.edu. 


\section{Identifying Nurses' Perceived Barriers to Mobilizing Patients on a Medical-Surgical Unit:}

A Quality Improvement Project

$$
\text { by }
$$

Jill Lukin
A Major Paper Submitted in Partial Fulfillment of the Requirements for the Degree of
Master of Science in Nursing
in

The School of Nursing

Rhode Island College 


\begin{abstract}
Researchers have found that low and moderate levels of mobility are independently associated with greater functional decline in activities of daily living (ADLs) at discharge (Zisberg et al., 2011) and that bedrest promotes declines in muscle mass and muscle strength (Coker et al., 2014; Dirks et al., 2016). The negative effects of low mobility and immobility are recognized by nurses, yet most acute care nurses do not prioritize the mobilization of their patients. Interventions to increase mobilization of hospitalized patients may be more effective if they are barrier targeted. The purpose of this quality improvement project was to identify nurses' perceived barriers to mobilizing patients on a medical-surgical unit in a community hospital. The project used a 26-item 5-point Likert style survey adapted from the Overall Provider Barriers survey; a validated selfadministered survey developed by Hoyer et al. (2015). The survey identified nurses' perceived barriers in three domains: knowledge, attitudes, and behaviors. A convenience sample of 28 nurses participated in the survey. Results demonstrated that three of the four most reported perceived barriers were in the behaviors domain, which assessed external factors that could influence the respondent's decision to mobilize or to not mobilize a patient. The highest barriers in the behaviors domain were inadequate staffing, lack of time, and patient resistance to being mobilized. The third highest overall barrier was the perception that increasing patient mobilization would be more work for nurses. This item was in the attitudes domain, which assessed the respondent's perception of patient safety, needs, and outcomes of mobilization and perception of available time, workload, and ability to mobilize patients. Results were consistent with previous studies that explored barriers to mobilization. Practical implications of the findings are discussed.
\end{abstract}




\section{Table of Contents}

Background/Statement of the Problem ......................................................................... 1

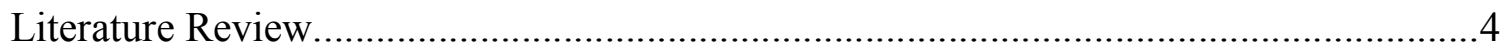

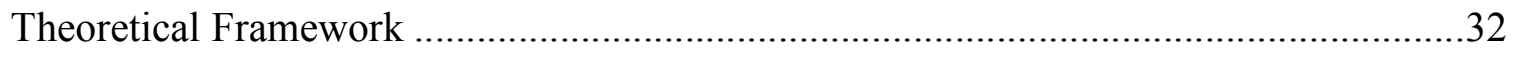

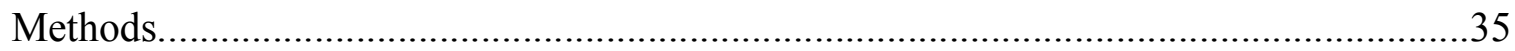

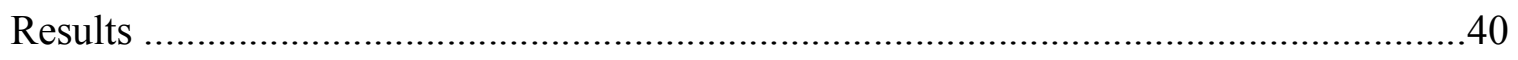

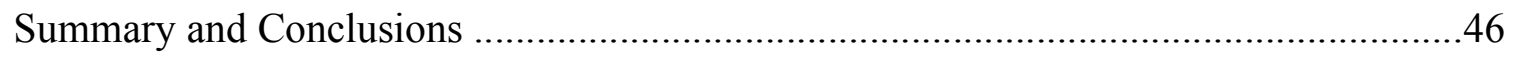

Recommendations and Implications for Advanced Nursing Practice ...........................49

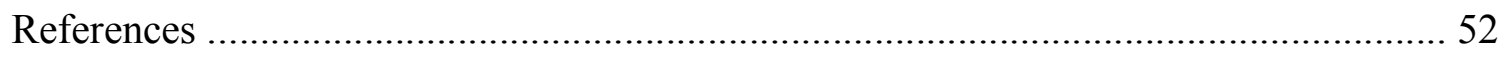

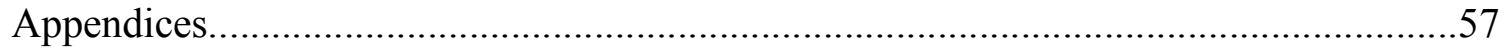


Identifying Nurses' Perceived Barriers to Mobilizing Patients on a Medical-Surgical Unit: A Quality Improvement Project

\section{Background/Statement of the Problem}

The negative effects of low mobility and immobility have been well documented in the literature, and the understanding that negative outcomes can prolong hospitalizations has remained undisputed, though more research is needed to support this link. Dirks et al. (2016) performed a small experimental study on ten young healthy males and found that the participants had substantial decline in muscle mass and function after 7 days of bedrest. Another study in Israel assessed the mobility rates of 525 older adults admitted to general medical inpatient units to determine the degree to which mobility affects functional status at discharge and found that low and moderate levels of mobility are independently associated with greater functional decline in activities of daily living (ADLs) at discharge (Zisberg et al., 2011). A third study in China identified 48 hours or more of bedrest time as an independent risk factor for developing a venous thromboembolism in patients undergoing gynecological surgery (Qu et al., 2015). Previous studies link negative outcomes, such as hospital-acquired pressure ulcers, thromboembolisms, and infections, with prolonged hospitalizations, especially in vulnerable older adults. In a retrospective observational study, Silke et al. (2011) found pressure ulcers, a well-known complication of immobility, to be an independent and significant predictor of a prolonged hospitalization stay for older adult patients. In an analysis of the impact of hospital acquired infection (HAI) in 155,891 trauma patients from a nationally representative database, length of stay (LOS) was found to be significantly higher in patients with HAI versus those without HAI (Silke et al., 2011). 
Most acute care nurses do not prioritize the mobilization of patients, despite understanding the benefits of mobilizing patients and the risks of not doing so. One prospective observational study in Australia found that medical and surgical patients spent $84 \%$ of the observed time in or on the bed, with no significant difference between older and younger adults (Mudge et al., 2016). Brown et al. (2009) found that hospitalized patients spent an average of 20 of every 24 hours in bed. The literature suggests that nurses' perceived and actual barriers contribute to low mobility and immobility. Brown et al. (2009) found that tubes, lines, and devices may limit mobility during hospitalization. The perception of increased workload for nurses and nurses not feeling confident in their ability to mobilize their patients have also been identified as barriers (Hoyer et al., 2015).

Nurses Improving Care for Healthsystem Elders (NICHE) is a nurse-driven program that helps healthcare organizations improve the care of older adults (NICHE, 2020). A major NICHE initiative is to increase mobility to reduce functional decline and improve outcomes. Sturdy Memorial Hospital (SMH), a community hospital in Attleboro, Massachusetts, has collaborated with Nurses Improving Care for Healthsystem Elders to make the targeted medical-surgical unit an Acute Care for Elders (ACE) unit. Using NICHE resources, SMH leaders have created new protocols and standards and have provided ongoing education regarding the care of older adults to the unit's nursing staff. The unit's leadership team has identified a goal of increasing nurse engagement in the mobilization of patients, particularly older adult patients, in alignment with the NICHE vision. Interventions targeted at the nurses' highest perceived barriers to mobilization may increase nurse engagement. The purpose of this quality improvement 
project was to identify nurses' perceived barriers to mobilizing patients on a medicalsurgical unit in a community hospital. A review of the literature will be discussed next. 


\section{Literature Review}

A literature review was conducted using the databases CINAHL Plus with Full Text and Google Scholar to examine the recent evidence surrounding hospitalized patients and mobility. Key search terms included immobility, low mobility, bedrest, effects, complications, risks, mobility, ambulation, length of stay, hospitalization, older adults, elderly, and geriatric in varying combinations. The searches were limited to articles published in English and from 2009 to 2020.

\section{Effects of Bedrest on Muscle and Physical Function}

Studies show that bedrest promotes declines in muscle mass and muscle strength (Coker et al., 2014; Dirks et al., 2016). A study of twelve healthy, physically active young males used dual-energy x-ray absorptiometry (DXA) to assess body composition 6-10 days before and immediately after 7 days of bedrest (Ringholm et al., 2011). On average, leg muscle mass was significantly reduced by $\sim 600 \mathrm{~g}$, but fiber type composition and size did not change significantly after bedrest. VO2 peak (maximal oxygen uptake) was $4 \%$ lower after bedrest when tested with an incremental bicycle test $(p \leq .05)$. Time to exhaustion after bedrest was not significantly reduced when tested with a one-legged knee extensor exercise test using a modified ergometer bicycle. Based on their findings, the authors suggested that total physical inactivity for 7 days alters exercise-induced gene responses and lowers metabolic capacity and metabolic regulation in skeletal muscles, potentially affecting functional abilities and skeletal muscles' adaptability to acute exercise.

Drummond et al. (2012) studied six healthy, older subjects 60-73 years old in an experimental study using DXA to determine body composition. After 7 days of bed rest, 
total lean mass and leg lean mass decreased significantly. In a separate controlled study, Drummond et al. (2013) also used DXA to measure body tissue composition in six community-dwelling, overall healthy, independent older adults aged 65-69 years old and found that the mean total body lean mass was significantly reduced by $\sim 1.6 \mathrm{~kg}$ after 7 days of bedrest. Approximately half of the loss $(\sim 0.8 \mathrm{~kg})$ was from leg lean mass. Diet was not controlled in this study.

An experimental study on 19 volunteers aged 60-85 with stable weight and no chronic diseases showed significantly decreased total lean mass (pre: $M=45.4 \mathrm{~kg}$, post: $M=44.6 \mathrm{~kg}$ ) and decreased lower extremity lean mass (pre: $M=14.4 \mathrm{~kg}$, post: $M=14.0$ $\mathrm{kg}$ ) after 10 days of bedrest (Coker et al., 2014). DXA was used to determine lean tissue. Pre- and post-bedrest VO2 peak was determined, and a significantly reduced VO2 peak was found after bedrest during maximal exercise using a bicycle ergometer (pre: $M=$ $21.5 \mathrm{l} / \mathrm{min}$, post: $M=18.6)$. Knee extension one repetition maximum (RM), isometric knee extension, and $60^{\circ}$ concentric knee extension were significantly reduced postbedrest. Knee extension one RM was significantly lower pre-bedrest in an undescribed subset of nine participants. A significant relationship was found between bedrest induced reduction in total lean mass and decrease in knee extension one RM. There was also a significant relationship between bedrest induced reduction in lower extremity lean mass and decrease in knee extension one RM. Other measures included floor transfer time, the time it took for a participant to move from a complete standing position to sitting, legs extended in front of the body and back up to standing, and the chair stand test, the time it took for the participant to stand up from a chair five times as quickly as possible without the use of their arms for assistance. Floor transfer time and chair stand time were 
significantly reduced post-bedrest (pre: $M=6.86$ seconds, post: $M=9.68$ seconds and pre: $M=8.41$ seconds, post: $M=9.43$ seconds, respectively). Participants were also timed while ascending and descending 10 steps. Power during stair ascent and descent was calculated as work divided by time in seconds. Stair ascent time, stair ascent power, and stair descent time were significantly reduced post-bedrest (pre: $M=4.37$ seconds, post: $M=5.29$ seconds; pre: $M=302.1$ newton-meters per second, post: $M=264.8$ newton-meters per second; and pre: $M=4.00$ seconds, post: $M=4.94$ seconds, respectively). Five-minute walk distance and walking speed were also significantly reduced post-bedrest (pre: $M=438.1$ meters, post: $M=405.5$ meters and pre: $M=1.46$ meters per second, post: $M=1.35$ meters per second, respectively). The authors hypothesized that unanticipated bed rest would lead to muscle loss that was closely related to decreased muscle strength and physical function. Though no significant relationships were found between total lean mass or lower extremity mass and changes in physical function, the authors concluded that significant reductions in total lean mass and lower extremity lean mass along with significant reductions in body mass index, muscle quality, and increased body fat percentage with bed rest occurred concurrently with decreased strength, physical function, and fitness level. They suggested that bedrest promotes detrimental changes to muscle strength and physical function.

In another experimental study of ten healthy young males, lean tissue mass, assessed by DXA before and after bedrest, declined significantly after 7 days of bedrest by $M=1.4 \mathrm{~kg}$ (Dirks et al., 2016). The lean tissue loss was mainly from the trunk and legs ( $M=1.0 \mathrm{~kg}$ and $M=0.28 \mathrm{~kg}$, respectively). Computed tomography also showed cross-sectional area decline in the thigh and lower back muscles $(M=2.2 \%$ and $M=$ 
$1.3 \%$, respectively). An incremental cycle ergometer was used to assess VO2 peak preand post-bedrest and showed a significant decline in the VO2 peak during maximal exercise $(M=6.4 \%)$. A significant decline in one RM leg press strength and leg extension strength was found ( $M=-7 \%$ and $M=-8 \%$, respectively). The authors concluded that short-term bedrest leads to declines in muscle mass and function.

\section{Risk of Functional Decline}

Zisberg et al. (2011) assessed the mobility rates of 525 adults 70 years old or older admitted to one of five general medical inpatient units in a 900-bed tertiary care teaching hospital in Israel to determine the degree to which mobility affects functional status at discharge. Patients who had had a stroke, were comatose, mechanically ventilated, or completely dependent in basic functions on admission or were admitted electively, had a LOS of 2 days or less, or were transferred from another unit were excluded. Trained research assistants, who were blinded to the study hypotheses, interviewed participants. The interviewers used the modified Barthel Index for ADLs, mobility level and ability to care for self, and Lawton and Brody's scale for instrumental ADLs, which assesses the ability to shop, prepare meals, use the telephone, and so forth, to assess participants' self-reported baseline functional status at 2 weeks prior to admission and self-reported baseline functional status at the time of admission. The Yale Physical Activity Survey was used to determine participants' self-reported level of mobility during the month prior to admission. Hospital mobility levels were assessed through daily interviews with a participant or surrogate using a modified mobility index previously developed. Interviews were conducted each day after the first 2 days, and up to three reports were averaged. Participants were given scores and categorized into low 
mobility (total bed rest or transferring from bed to chair up to twice a day), moderate mobility (ambulation inside the room only), or high mobility (ambulation at least once a day outside the room in addition to mobility inside the room). Additional interviews were conducted at discharge and at 1 month following discharge. In contrast to other studies regarding the mobility level of hospitalized older adults, $65 \%$ of participants were highly mobile during their entire hospital stay, and $16 \%$ of participants were moderately mobile. Of the $19 \%$ of participants who had low mobility levels during their stay, $86 \%$ had declined in ADLs at discharge, and 73\% had declined in ADLs at 1-month follow-up ( $p<$ .001). Adjusted odds ratios showed significantly higher odds of functional decline at discharge and at 1-month follow-up for low and moderately mobile patients than for highly mobile patients.

\section{Time Spent in Bed versus Mobilizing}

A number of studies have examined the prevalence of low mobility in different medical-surgical settings. One study by Brown et al. (2009) found that hospitalized patients spent an average of 20 of every 24 hours in bed. The study's objective was to examine the proportion of time spent in three levels of mobility (lying, sitting, and standing or walking). Participants wore wireless mobility monitors validated to measure levels of hospital mobility. The monitor was worn on the leg for 7 days or until the patient was discharged. None of the selected patients spent their entire measured hospital stay in bed. On average, the cohort spent $83.3+/-12.2 \%$ of their time lying in the bed, and $33 \%$ of patients spent more than $90 \%$ of their hospital stay in bed. Patients spent an average of 3.1 hours sitting and 55 minutes standing or walking per day. Power analysis was not conducted. The study included a consecutive sample of 45 male patients aged 65 
and older admitted to the medical wards of a Veterans Affairs Medical Center. Some of the inclusion criteria were chosen with the intent to select participants who would be more likely to be ambulatory during hospitalization, and though this does not best represent the population, it shows that low mobility is a problem even among those hospitalized patients who are capable and likely easiest to mobilize.

In a similar study at the Copenhagen University Hospital in Denmark, 24-hour mobility was measured in 42 patients who were able to walk unassisted on admission and compared to six patients who were unable to walk on admission in a prospective cohort study (Pedersen et al., 2013). The six patients who were unable to walk were included as an immobility reference. Participants were acute medical inpatients 65 years or older admitted from home with at least one comorbidity. Two randomly selected patients were enrolled in the study daily. Validated wireless accelerometers worn 24 hours a day above the patella and above the ankle joint were used to assess mobility level from within 48 hours of admission to discharge. Only patient days with more than 18 hours of measuring were included, and no more than 10 days were recorded. The monitors sampled every second, measuring horizontal position, vertical position, and depth with respect to gravity, which were used in an algorithm to determine patient positions. The results showed that the acutely admitted older medical inpatients who were able to walk on admission spent an average of 17 hours in bed, 5.1 hours sitting, and 1.1 hours standing and/or walking per day while hospitalized compared to the non-ambulatory patients who spent 22.6 hours in bed, 1.0 hours sitting, and 0.2 hours standing and/or walking $(p=.0002, p=.0006$, and $p=.0008$, respectively). The study was limited by being unable to discriminate between standing and walking and because it did not 
evaluate the level of mobility of patients who could walk with personal assistance; however, it is reasonable to assume that these patients spend even less time mobilizing than patients who can walk independently in the hospital.

Another study by Fisher et al. (2011) describes the amount and patterns of ambulatory activity in hospitalized older adults over consecutive hospital days and found that, on average, older adult hospitalized patients spent $95.9 \%$ of time being inactive, defined as not engaged in any ambulatory activity. The sample included 239 patients aged 65 and older admitted to the Acute Care for Elders (ACE) unit at a university teaching hospital. The study participants wore a step activity monitor (SAM), which was placed on the day of admission and worn until discharge. Patients who had a contraindication to wearing the SAM were excluded, as were patients with a primary orthopedic surgical diagnosis. All other patients during the four consecutive month data collection period were eligible for inclusion in the study. The monitors recorded the total number of steps per 24-hour day, and the mean daily steps were calculated based on number of days the monitor was worn. The SAMs have been shown to have $98 \%$ accuracy in various clinical populations. The study showed that on $50 \%$ of observation days, patients took fewer than 300 steps, on $12.9 \%$ of observation days, patients took no steps, and on only $1 \%$ of days, patients took more than 4,100 steps. Prolonged LOS (seven or more days) was found to be one of the factors associated with the lowest ambulatory activity. Length of stay $(p=.03)$, age $(p=.05)$, preadmission mobility status ( $p<.001)$, prior ADLs limitations $(p<.001)$, history of falls $(p=.002)$, confusion or delirium during the admission assessment $(p=.02)$, physician's admitting activity orders $(p=.05)$, and a physical therapy consultation during hospitalization $(p<.001)$ showed 
statistically significant differences in mean steps per day. Age 75 and older, mobility impairment prior to admission, history of falls, delirium, a physician's bed rest order at admission, and having a physical therapy consultation at any point during the hospital stay were all associated with fewer mean daily steps during hospitalization. A subset of patients who took fewer than 500 daily steps (the approximate median value for daily steps, for at least 5 consecutive 24-hour days $(\mathrm{n}=27)$ was found to be significantly more likely to have a prior history of falls $(41.6 \%)$ than all other patients $(18.5 \% ; p=.02)$. The patients who took fewer than 500 daily steps were more likely to have preadmission limitations in basic ADLs ( $58.3 \%$ vs $32.6 \% ; p=.03)$ and significantly less likely to be classified as independent ambulators prior to admission $(20.8 \%$ vs $61.9 \% ; p<.001)$. Because the patients studied were on an Acute Care for Elders (ACE) unit, generalizability may be limited with increased selection bias. Though this setting is a limitation of the study and might not be representative of the experience of older adults admitted to a traditional hospital ward, it is important to note that the findings likely underrepresent the scope of the problem of immobility in older hospitalized adults. Additionally, the awareness of activity being observed due to participants having to wear the monitors on the leg could have introduced reactivity and performance bias since neither the participants nor staff could be blinded. This limitation is more likely to have influenced participants and caregivers to increase mobility, making it possible that hospitalized older adults spend even more time in bed than the findings indicate and underscoring the need for additional studies.

A small observational study by Doherty-King et al. (2014) evaluated the frequency and duration of nursing care activity related to mobilizing patients older than 
65 years of age on two medical-surgical units at an 81-bed veterans hospital and on four medical units at a 485-bed academic teaching hospital $(N=47)$. The researchers also sought to determine if the mobility events were patient or nurse initiated. A time-andmotion design was used to observe 15 nurses during their 8-hour shifts for a total of 43 shifts. Observers collected the frequency and duration of six types of mobility events: standing, transferring, walking to the bathroom, walking from the bathroom, walking within the patient room, and walking in the hallways. They also recorded if the patient or the nurse initiated the mobility activity. To avoid performance bias, nurses were told that all patient care activities were being recorded. Of the 47 patients, $31.9 \%$ had no mobility events during their observation period. The highest mean frequencies were for standing and transferring events $(M=0.7$ and 0.5 per observation period, respectively). The median was zero for all six types of activities, indicating that for each type of activity, over half of the patients did not have an activity event observed. The nurse initiated only two of the eleven walking in the hallway events and four of the twenty walking in the room events. Walking in the hallway had the highest mean duration ( $M=1.8$ minutes).

Data from all the patients was compared to a subgroup of 16 dependent patients able to ambulate but needing human assistance (Doherty-King et al., 2014). No mobility events occurred in $31.3 \%$ of the dependent subgroup during the observation period. Transferring was the most frequently observed activity among dependent patients $(M=$ 0.8 per observation period). Walking in the hallway had the highest mean duration among dependent patients ( $M=0.5$ minutes). Transferring was the only activity that had a median greater than zero (0.2 minutes) among dependent patients. All dependent patients' ambulation events were initiated by the patient. Observers' field notes revealed 
that standing and transferring events in all patients always occurred after medical procedure-related activities, such as getting up for meals and obtaining a weight. Between 7 a.m. and 10 a.m. and 3 p.m. and 6 p.m., nurses were always noted to be performing activities related to giving and receiving report from nurses and nursing assistants, assessing their patients, and administering medications. The sample size was small, and patients may have mobilized with other staff members on the observation days, limiting the study's findings. The authors acknowledged a lack of investigation into potential confounding factors and a potential Hawthorne effect as limitations. Given the limitations in these studies, it seems reasonable to expect similar or worse outcomes in other groups of hospitalized older adults.

Mudge et al. (2016) conducted a prospective observational study using crosssectional sampling from a 26-bed general medical ward, a 30-bed oncology ward, and a 24-bed vascular surgical ward in a 900-bed tertiary teaching hospital in Australia. Only patients receiving end-of-life care were excluded from the study. Trained physiotherapy students observed and categorized patients as lying in bed, sitting in or on the bed, sitting on a chair, standing, actively wheeling, or walking. Participants were observed during four observation periods for two mornings (1000-1400) and two afternoons (1400-1800) within 10 days. Participants' activity levels were recorded for 2-minute increments before observers moved to the next room, and the sequence was repeated in the same order for a four-hour period with one 15 -minute break, providing 12 to 17 observations per participant for each four-hour period. Of 3272 valid observations (at least 2 hours in one or more observation period) available for physical activity in 132 patients, mean $57.4 \%$ of observed time was spent lying in bed, $33.6 \%$ sitting on the bed or chair, and $9.0 \%$ 
standing, walking, or wheeling. Across all observation periods, $29.5 \%$ of participants were never observed to be standing, walking, or wheeling, and 7.6\% were in bed during each observation. On the medical and vascular surgical units, $84 \%$ of the time was spent in or on the bed, and $16 \%$ of the time was spent sitting in a chair or in active upright postures. Vascular surgical patients were observed lying flat in bed two-thirds of the time, and time spent standing, walking, or wheeling was significantly lower on the surgical unit $(p=.015)$. Vascular surgical patients were observed standing, walking, or wheeling $6.5 \pm 9.4 \%$ of the time compared to $8.2 \pm 8.4 \%$ in medical patients and $11.4 \pm$ $9.7 \%$ in oncology patients. There was no significant difference in the amount of time patients younger than 65 years of age and patients 65 years of age and older spent in active upright positions. During the auditing period, the oncology and medical wards were involved in the intervention stage of a quality improvement project to improve early mobility, while the vascular surgical ward was in the information-gathering stage, limiting the study's comparison findings.

\section{Mobility Protocols}

Padula et al. (2009) implemented a nurse-driven mobility protocol in which the registered nurse (RN) is expected to question orders for bed rest and routinely evaluate the necessity of obstacles to mobility, while certified nursing assistants (CNAs) are expected to ambulate patients three to four times per day, assist patients to the chair for meals, and assist older adults to the bathroom or commode for toileting. The study's purpose was to determine the impact of a nurse-driven mobility protocol on functional decline. Sample size statistics were calculated prior to data collection to determine adequate power to detect differences between the groups and supported a total sample 
size equal to 50. Two units were compared and were equal in size and had similar patient populations and nursing staff composition. A total of 50 adults 60 years or older who were admitted with medical diagnoses to one of the two units were recruited for the study $(\mathrm{n}=25$ each). To be eligible, participants were required to have a LOS of at least 3 days, understand English, be cognitively intact or have a significant other who could participate, and be without a physical impairment that would significantly limit their ability to mobilize.

For data collection, a demographic data collection sheet was used along with the modified Barthel Index, the Get Up and Go test, and the Mini Mental Status Exam. Descriptive statistics for study variables were completed and comparisons between the groups on baseline characteristics were examined. The findings showed that modified Barthel Index preadmission scores, which reflect subjects' perceptions of functioning 2 weeks prior to admission, were significantly higher than admission scores for both groups. Discharge modified Barthel Index scores significantly improved by +11.5 ( $p=$ .05 ) from the admission baseline for the treatment group, and the control group improved by 6.9 , which was not statistically significant. The control group showed a statistically significant decrease in function between preadmission (87.08) to discharge $(71.08 ; p=$ .006), and while the treatment group scores were lower at discharge (80.60) than at preadmission (88.76), the differences were not statistically significant. The Up and Go test scores improved slightly from admission to discharge for both groups but were not statistically significant. Treatment group subjects were out of bed to chair significantly less often (treatment 5.9 times, control 8.7 times; $p=.05$ ). Treatment group subjects ambulated in the hallway earlier (treatment 2.7 days, control 4.9 days; $p=.007$ ). 
Treatment group subjects ambulated in the hall more often, but the difference was not statistically significant (treatment 3.12 times, control 2.44). Finally, the treatment group had significantly shorter LOS than the control group (treatment 4.96 days, control 8.72 days; $p<.001)$

The researchers acknowledge that the insignificant but lower functional level of the control group could have contributed to increased LOS and/or later ambulation (Padula, et al., 2009). Additionally, due to control group data inconsistent with the literature, the authors postulated that the presence of a unit-based advanced practice nurse with responsibilities for clinical outcomes on the unit, unique to the control group unit, may have affected the higher than expected mobility outcomes. Although further research is needed to corroborate the study's findings, mobilizing hospitalized patients suggests improved outcomes for older adults.

A quasi-experimental design by Drolet et al. (2013) was used before and after a nurse-driven mobility protocol was implemented in a 16-bed medical-surgical intensive care unit (ICU) and 26-bed intermediate care unit (IMCU) at a 1,313-bed community hospital. A mobility algorithm was developed by a physical therapist and embedded in an order set, and a ventilator weaning set was created to include pain and sedation management protocols. Posters were placed on the units to remind staff about the study, and verbal presentations and self-learning packets were given to nurses and patient care technicians. Advanced practice nurses and physical therapists educated them on patient exclusion criteria, the mobility algorithm, and the use of gait belts for mobilizing patients. Little improvement was noted in ambulation until a daily ambulation status report was given to the nurses, providing real-time data about the patient's LOS and how much each 
patient had ambulated on a given day. During the pre-implementation phase, $6.2 \%$ and $15.5 \%$ of ICU and IMCU patients ambulated within 72 hours of hospital admission compared to $20.2 \%$ and $71.8 \%(p<.001)$, respectively, during the 6 months following implementation of the mobility protocol. Frequency of ambulation was not recorded. The study suggests that mobility protocols can significantly increase the number of patients who are mobilized early in their hospitalizations.

\section{Barriers to Patient Mobilization}

The older adults who participated in a nurse-driven mobility protocol were shown to have a reduced LOS (Padula et al., 2009); although, in order to effectively implement practice changes in most settings, barriers first need to be understood. In one study, barriers to mobilization included availability of CNAs and high unit activity (DohertyKing \& Bowers, 2011). The authors developed a conceptual model based on nurses' discussions of how they decided whether, when, and how to ambulate patients. The model reflects the findings that nurses mobilize patients to prevent complications, monitor progress, and to comply with written orders or unit expectations.

It is suggested that patient, nurse, and organizational characteristics influence nurses' decisions about how to mobilize patients (Doherty-King \& Bowers, 2011). Circumstances, characterized by the availability of resources needed to safely mobilize patients, and the potential for injury to the patient or nurse during patient mobilization are also influential factors. Clear expectations for ambulation at the unit level was found to have the most significant impact on whether nurses ambulated patients, specifically when combined with increased accountability and consequences for not mobilizing patients. 
Another study found that urinary catheters, intravenous lines, and oxygen may limit mobility during hospitalization (Brown et al., 2009).

Attitudes that increasing patient mobilization is more work for nurses and nurses not feeling confident in their ability to mobilize their patients have also been identified as barriers (Hoyer et al., 2015). Such barriers are found to be higher for staff with less experience, according to a recent study aimed at examining providers' knowledge, attitudes, and behaviors regarding patient mobility. The Overall Provider Barriers scale is a validated self-administered survey developed by Hoyer et al. (2015) to assess the perceived barriers of direct patient care providers. Published guidelines and previous research were used to develop the survey, further refined through pilot testing by providers of multiple disciplines. Barriers in three domains, knowledge (four items), attitudes (nine items), and behaviors (13 items) form subscales within the Overall Provider Barriers scale. The survey also collects data on provider discipline and years of clinical experience in the hospital. Survey instructions define mobilizing patients as "getting a patient out of bed (e.g., sitting out of bed, toileting at bedside or to a bathroom, standing, and ambulation)" (Hoyer et al., 2015, Survey Development section). The knowledge subscale assesses the provider's perception of training and education relating to mobilizing patients and appropriate consultation to rehabilitation services. The attitudes subscale assesses the provider's perception of patient safety, needs, and outcomes of mobilization, along with the perception of available time, workload, and ability to mobilize patients. The behaviors subscale assesses external factors that may influence the provider's decision to mobilize or to not mobilize a patient. Responses to statements on the Overall Provider Barriers survey are chosen from a 5-point Likert scale 
to indicate degree of agreement (strongly disagree, disagree, neutral, agree, and strongly agree). The key variables, which are the perceived barriers in the three domains (knowledge, attitudes, and behaviors), are based on a conceptual framework to understand barriers to provider adherence to practice guidelines.

A convenience sample of 120 full-time nurses and physical and rehabilitation therapists (rehabilitation therapists, 38; nurses, 82), who predominantly worked on six targeted general medicine units, in two different community hospitals, on the day shift, were surveyed. Nurses, compared to rehabilitation therapists, were found to have significantly higher barrier scores $(\mathrm{P}<.001)$ on the Overall Provider Barriers scale and all three sub-scales. An increase in five years of experience was shown to have a small but significant association with lower Overall Provider Barriers $(p=.02)$ and knowledge $(p=.009)$ and attitudes $(p=.04)$ subscale scores. There were no significant statistical interactions between hospital site, discipline, and years of experience. The item with a mean score of 4 , reflecting the highest perceived barrier by nurses, was that "increasing mobilization of my inpatients will be more work for nurses" (Hoyer et al., 2015, p. 6, para. 4). Inadequate nurse-to-patient staffing was also identified as a barrier $(M=2.7$ in response to staffing being adequate). Receiving training in mobilizing patients was highly correlated with confidence in mobilizing patients $(r=0.65, p<.0001)$, and most nurses disagreed with not feeling confident mobilizing their patients $(M=2.3$ in response to not feeling confident). Mean scores were 3.7-4.0 for educating inpatients to increase their activity and for knowledge of adequate training and appropriate referral to rehabilitative services. Most nurses $(M=4.2)$ believed that inpatients who are mobilized at least three times daily will have better outcomes. 
An adapted version of the Overall Provider Barrier scale was used in a descriptive correlation study conducted by Dermody et al. (2017) in two community hospitals, and $85(N=101)$ nurses who worked more than 20 hours a week on various non-critical care units responded to surveys. Two questions that surveyed the nurse's individual and the employer's prioritization of promoting mobility in hospitalized older adults and one question that surveyed the nurse's knowledge of assessing the lower length strength of hospitalized older adults were added to the original survey. Some nurses (18.8\%) reported that they did not receive adequate training on safe patient mobilization, and $19 \%$ of nurses believed that a rehabilitative therapist should be the primary care provider to mobilize their patients. Most nurses (84\%) reported promotion of mobility of hospitalized older adults as a priority, and $94 \%$ of nurses believed that their patients who are mobilized at least three times a day will have better outcomes. Forty-four percent of nurses believed that promoting mobility in hospitalized older adults is a priority for their organization. Inadequate staffing was identified as a barrier by $61 \%$ of nurses, and $89 \%$ of nurses believed that increasing patient mobilization would be more work for them. Risk for self-injury was identified by $54 \%$ of nurses, lack of time by $47 \%$ of nurses, and patient resistance to being mobilized by $48 \%$ of nurses. Other barriers included believing that their patients were too sick to be mobilized (19\%), lack of confidence in their ability to mobilize their patients (13\%), and feeling unsure of when it is safe to mobilize their patients $(10 \%)$. Nurses with less than five years of experience had significantly lower perceptions of receipt of adequate training and appropriate referral to rehabilitative services $(p=.004$ and $p=.047$, respectively). Less experienced nurses showed significantly more confidence in mobilizing patients but were less likely to prioritize 
patient mobility ( $p=.009$ and $p=.024$, respectively). Less experienced nurses were also significantly less likely to perceive that patients who can be mobilized have appropriate physician orders and more likely to perceive lack of time as a barrier to mobilizing patients during a shift ( $p=.053$ and $p=.048$, respectively).

\section{Barrier-Targeted Interventions}

Researchers at an academic medical center in northern Israel used a modified version of the Overall Provider Barrier scale developed by Hoyer et al. (2015) to survey 116 nurses, nurse's aides, physical therapists, and medical doctors working in two internal medical units at an academic medical center (Zisberg et al., 2018). In addition to surveying staff, four focus groups were held to determine strategies to adapt existing interventions and policies to the organization's needs to improve mobility. As part of the comprehensive, multiphase prospective cohort study, researchers also surveyed 203 older adults hospitalized on the two internal medical units to determine their attitudes toward in-hospital mobility and their intention to be mobile during their hospitalization using a reliable and valid 6-item scale. Their level of mobility was assessed by daily step count using accelerometers worn from 24 hours to 72 hours. The older adult participants' daily measured steps were linked to functional outcomes, which was used to determine 900 steps per day as the desired mobility level that would prevent clinically meaningful functional declines.

On a scale of $1-4$, nurses were found to have poor knowledge $(M=2.45 \pm .36$ $\mathrm{SD})$, negative attitude $(2.53 \pm .48)$, and higher barriers regarding in-hospital mobility compared to physical therapists ( $3.57 \pm .35$ and $3.00 \pm .45$, respectively) (Zisberg et al., 2018). The highest perceived barriers among all the healthcare providers surveyed were 
inadequate staffing $(2.1 \pm .8)$, lack of equipment $(1.6 \pm .9)$, lack of regular interdisciplinary discussion of patient's mobility $(1.8 \pm .8)$, and except for physical therapists, inadequate training on safe patient mobilization ( $2.2 \pm 1.0)$. Based on discussions from the focus groups, interventions were developed and included an online educational module partially adapted from the Mobilisation of Vulnerable Elders in Ontario (MOVE ON) project, bedside training by physical therapists, electronic reports of mobility assessments, mandatory standardized documentation, walking trails, and the purchase of extra walkers. Patient and family brochures and posters, adapted from MOVE ON, provided recommendations for maintaining mobility. Nursing staff were introduced to all the interventions and new workflows. Physical therapists trained the nursing staff at the bedside on how to safely mobilize patients. Electronic documentation was reviewed daily, and weekly discussions of the mobility protocol implementation were held. Staff were re-surveyed $(n=83) 3$ months after the new protocol was initiated, and knowledge $(p=.005)$, behavior $(p<.001)$, and attitudes $(p=.035)$ were found to be significantly improved, most notably among nursing aides and nurses.

Most patients surveyed during the pre-implementation period had positive attitudes regarding in-hospital mobility $(3.36 \pm .97$, on $1-5$ scale, with $65 \%$ expressing above neutral values), but $46.5 \%$ agreed to some extent with the statement "I need to stay in the bed when I'm sick," and 39.6\% disagreed with the statement that "walking in the hospital will help me maintain my pre-hospital function" (Zisberg et al., 2018). After controlling for objective mobility ability on admission, severity of illness, LOS, cognitive function, age, and premorbid mobility level, the odds of low mobility were found to be 3.22 (95\% CI: 1.24-8.44) times higher for patients with negative to neutral attitudes than 
for those with positive attitudes toward in-hospital mobility. Negative to neutral attitudes were significantly related to walking less than 900 steps.

When patients were surveyed three months after implementation, the percent of patients who reported that they received walking encouragement during their stay increased significantly from $17 \%$ to $84 \%(p<.001)$ (Zisberg et al., 2018). Positive responses to the phrase "I believe that increasing in-hospital mobility will improve my recovery" increased significantly from $48 \%$ to $57 \%(p<.001)$. Patient steps increased significantly from 1243 steps versus 2356 steps per day after protocol implementation ( $p$ $<.001$ ), and the number of patients who walked more than 900 steps per day after the protocol implementation increased significantly from $61 \%$ to $87 \%(p<.001)$.

According to the Institute of Medicine, a systems approach is necessary to develop innovative and sustainable change. King et al. (2016) acknowledged that current approaches to implement interventions to increase ambulation in hospitalized older adults typically focus on a single barrier, despite the presence of multiple other system and personal barriers. The failure to address multiple barriers simultaneously may thwart efforts to improve functional outcomes for hospitalized older adults. King et al. (2016) conducted a pilot study to evaluate the implementation of Mobilizing Older Adult patients via a Nurse-driven intervention (MOVIN), a systems-based intervention targeted at barriers to nurse-initiated patient ambulation, on a 26-bed adult general medical unit at a 648-bed tertiary academic hospital. A mixed quantitative and qualitative study design was used to evaluate the outcomes related to the implementation of MOVIN, an investigator-developed, nurse-driven intervention comprising five components: psychomotor skills training to increase RNs' self-efficacy in determining whether 
it is safe for patients to ambulate and in helping older adults to ambulate, communication tools (e.g., ambulation white boards) to improve information sharing between nursing staff (CNAs and RNs) and other healthcare providers (PTs and physicians) about how well patients ambulate, ambulation pathways that are interesting and provide visual markers to measure distances that patients ambulate, ambulation resources (human and equipment) to maximize opportunity for patient ambulation, and ambulation culture to establish nurse ownership and sustainability of patient ambulation. (King et al., 2016, p. 2089, para. 2)

MOVIN was developed by the researchers based on prior research on identifying barriers to patient ambulation. A quasi-experimental, one group pretest-posttest design was used to evaluate three objective measures of nurse practice change: ambulation frequency, ambulation distance, and numeric documentation of ambulation distance (percentage of documentation in the electronic medical record [EMR] in which nursing staff enter a numeric value of distance rather than text descriptors). Data reports were generated weekly. Focus group interviews and a single individual interview with semi-structured interview questions were conducted after the intervention to evaluate the five components of MOVIN. All 32 RNs and 10 CNAs on the study unit were exposed to the interventions, 18 RNs completed the psychomotor skills training, 10 RNs and five CNAs participated in focus group interviews, and one RN participated in an individual interview. Results of the nurse-driven ambulation protocol pilot demonstrated increased ambulation frequency, increased ambulation distance, and increased numeric documentation. There were 150 more occurrences of ambulation between the end of the preintervention phase and the intervention phase $(p=.001)$. Ambulation distance was 
significantly greater during the intervention phase than during the preintervention phase $(p=.01)$. There was a significant increase in numeric documentation during the intervention phase than in the preintervention phase $(p=.04)$. The focus group and individual interviews indicated high acceptance and perceived benefit of all five components of MOVIN. A shift in unit culture toward high nursing staff engagement, ownership, and confidence in ambulation decision-making were also observed.

In Canada, a large-scale quasi-experimental interrupted time series design was used to evaluate the impact of an early mobilization protocol on patient mobilization during preintervention, during intervention, and during post-intervention (Liu et al., 2017). A secondary outcome was LOS. The final sample included 12490 patients 65 years and older admitted to inpatient medical units in eleven university-affiliated hospitals in Ontario and excluded patients on palliative care or on bed rest. Each hospital had an implementation team that included a physician leader, education coordinator, and a research coordinator. A multi-component intervention included interprofessional education and educational tools; resources, such as education modules, checklists, and mobility algorithms; and implementation coaching from the central MOVE ON team. Additional strategies, such as reminders, local opinion leaders, and patient/caregiver education materials chosen based on local context, were also part of the intervention. The local teams had access to an online community of practice and participated in monthly teleconferences. Each local team worked with coaches to choose intervention strategies based on local barriers and facilitators that were identified through focus groups with multiple caregivers. Mobilization status of patients was measured by twice-weekly, random weekday visual audits three times daily. "Mobilized" was defined as being out of 
bed. Visual audits were evaluated to have high inter-rater agreement. The daily mobility recorded during the three audits per day was summarized as the proportion of patients mobilized each day, and the proportion was averaged over the 2 audit days per week. That proportion was averaged across all eleven hospitals for an estimate of overall daily mobility. Based on the 115025 observations from 12490 patients, the authors found that $10.56 \%$ more patients were mobilized per day post-intervention compared to preintervention, a statistically significant difference $(p<.001)$. During the intervention, $3.12 \%$ more patients were found to be mobilized per day than in the preintervention phase, though this was not a statistically significant difference. Compared to the intervention period, $4.28 \%$ more patients were mobilized during the post-intervention period $(p=.005)$. The results suggested that implementing interventions tailored to a specific unit can increase the amount of time hospitalized older adult patients spend out of bed. During the intervention period, the median LOS significantly decreased by 3.45 days on average across all sites $(p=.0356)$, but the median LOS increased during the intervention compared to the preintervention period, which was not statistically significant. The median length of stay during the post-intervention period was 6.1 days shorter than the preintervention period $(p=.015)$. The generalizability of the outcomes related to LOS are limited by the lack of research on confounding factors.

Holroyd-Leduc et al. (2019) conducted a replication study of the 2017 Liu et al. study to evaluate the impact of mobilization interventions on seven units in four community hospitals in Alberta, Canada. A pragmatic, quasi-experimental unblinded interrupted time series design was used to determine the rate of mobilization at preintervention, during the intervention, and at post-intervention. The study was 
conducted on one long-term/restorative care unit, one general medicine unit, one surgical unit, one geriatric rehabilitation unit, one short stay surgical unit, and two intensive care units. Patients 65 years and older were included. Patients on palliative care or bed rest were excluded. Each site created local implementation teams that included a physician lead, research coordinator, nursing education coordinator, and one to two other key staff members who engaged in monthly steering committee meetings with members of a centralized implementation support team. Staff on all participating units completed a readiness assessment survey and an online barriers and facilitators assessment tool during the preintervention phase. Implementation activities were selected using the MOVE ON online portal of resources. Activities were tailored to the local context of each site. All sites used champions, whiteboards, log sheets, audit feedback, educational meetings, bedside education, and educational materials. Site implementation teams completed a sustainability survey during the pre- and post-intervention phases, and findings were used as an implementation support tool. Visual audits were conducted three times a day for 2 days each week during each phase for a total of 42840 mobility audits from 3601 patients. Trained research assistants recorded types of mobility, categorized as standing/walking with assistance, standing/walking supervised, standing/walking independently, and immobile. "Immobile" patients included those lying in bed, sitting upright in bed, sitting in a chair, and those who could not be observed. The proportion of patients mobilized was averaged over the 2 audit days and represented average daily mobilization for a given week, which was aggregated across all sites to provide an overall estimate. Significantly more (an estimated 6\%) out of bed activities were recorded at the end of the intervention period compared to the end of the preintervention period $(\mathrm{p}=$ 
.0173). A decrease in mobilization rate was noted post-intervention. An estimated $-5 \%$ fewer out of bed activities were noted at the end of the post-implementation phase compared to during the intervention phase. The findings suggest that a barrier-targeted mobility protocol can increase in-hospital mobility short-term, but more research is needed on creating lasting change.

Secondary outcomes of the study by Holroyd-Leduc et al. (2019) included LOS and perceptions of the MOVE intervention from site implementation teams, participating unit staff, and patient/family members. No significant change in LOS was shown. Twenty-nine staff members (including 8 nurses) and 45 patients/family members completed anonymous exit surveys to evaluate perceptions. Most of the staff respondents reported staff education (72\%) and educational materials (76\%) as being effective in increasing their knowledge. Fifty-one percent of patients reported that education activities and materials were effective in increasing their knowledge about the importance of mobilizing during their hospitalization, and $40 \%$ of patients reported that educational activities were effective in helping them mobilize. Fifty-nine percent of patients reported that staff always or frequently encouraged them to perform more out of bed activities. Nine site implementation team members participated in semi-structured phone interviews. Participants commonly identified early education sessions, regular, dedicated time with unit staff to discuss implementation, consistent communication between central coaches and unit staff, and the importance of creating a culture change as key requirements for informing, engaging, and supporting staff. During the preintervention phase, strong senior leadership engagement and adaptability were identified as strengths. Lack of infrastructure, lack of staff involvement, and lack of training to sustain the new 
processes were identified as challenges. Clinical leadership engagement was found to be a common strength during the post-intervention phase. Lack of senior leadership engagement was identified as a challenge. Limitations of this study include the lack of consideration of external factors that may have impacted LOS and the variability of the study sites and selected interventions.

Jones et al. (2019) surveyed 104 medical-surgical RNs across five units in a 547bed community hospital about their belief of knowledge, confidence, attitude, commitment, and barriers to mobility. In response to the needs identified in the survey, 480 nurses were provided classroom education and competency validation of their use of the Bedside Mobility Assessment Tool (BMAT), which was selected to assess mobility and guide safe patient handling. CNAs were provided "modified" classroom training (not detailed). Standardized interventions in line with the BMAT were added to the electronic health record (EHR) and incorporated into handoffs, whiteboard communication, and standards of care. RNs performed and documented the BMAT assessments every shift. An "activity per nursing assessment" order was created as the standard mobility order for medical-surgical inpatients. Strict bed rest orders were programmed to automatically expire within 24 hours, unless a physician provided supportive clinical rationale for otherwise. Standardized criteria for physical therapy consultations were also added to the EHR. Additionally, staff were engaged in creating awareness and excitement about the project, a walker and gait belt were placed in every patient room, and distance markers were placed in the hallways. A mobility coordinator was hired and implemented "daily rounding and auditing, real-time feedback for individual performance, data collection, mobility training for newly hired staff, and management of mobility and lift equipment" 
(Jones et al., 2019, p 22). A retrospective chart audit was conducted on all medicalsurgical admissions during the year prior to the intervention to obtain baseline data for documentation of patient mobilization by nursing staff and on complete bed rest orders in the EHR. These data were then collected for one-year post-intervention. Ambulation to the bathroom was not included in the data collection. Random audits were conducted on 90 patient charts to measure the appropriateness of physical therapy orders at baseline and every 3 months for one year. Results indicated that there was a significant overall increase in nurse-led patient mobilizations in the post-implementation sample of 13673 patients compared to the pre-implementation sample of 14081 patients $(M=2.45 \mathrm{vs} 1.75$, respectively; $p<.01)$. Each of the individual units showed a significant difference in preand post-implementation nurse-led mobilizations $(p<.01)$. Complete bed rest orders were significantly reduced overall from $21 \%$ in the pre-implementation sample of 2921 patients to $9 \%$ in the post-implementation sample of 1258 patients $(p<.01)$. Complete bed rest orders were significantly reduced on individual units $(p<.01)$. Preimplementation, $22 \%$ of physical therapy orders were found to be inappropriate. Inappropriate physical therapy orders were reduced to $4 \%$ at 6 months and $8 \%$ at oneyear post-implementation. Sample sizes and statistical significance were not discussed.

Based on the literature, initiation of a mobility protocol may decrease barriers related to inadequate staffing and lack of confidence in nurses' abilities to mobilize patients and decrease nurses' workloads. Additionally, it may result in improved communication of documentation of activity level, distance, and frequency, as well as increase the visibility of mobilization, thereby leading to an altered unit culture. Consequently, it is the hope that the culture becomes one that views patient mobilization 
as the norm instead of the exception. Current literature on the prevalence and effects of immobility is lacking, as are studies focusing on the outcomes of interventions to increase mobility in hospitalized older adults. Further research is needed to examine barriers to inpatient mobilization and outcomes related to barrier-targeted mobilization protocols and initiatives. The purpose of this quality improvement project was to identify nurses' perceived barriers to mobilizing patients on a medical-surgical unit in a community hospital. Next, the theoretical framework will be discussed. 


\section{Theoretical Framework}

The theoretical framework used to guide this quality improvement project is the Theory of Planned Behavior (TPB), developed in 1985 by Icek Ajzen. The framework is an extension of the Theory of Reasoned Action (TRA), which was developed in 1975 by Icek Ajzen and Martin Fishbein and examines how a person's beliefs influence their behaviors. The TRA is designed to predict the many day-to-day behaviors that are under volitional control and to aid in understanding those behaviors' psychological determinants (Ajzen, 1985). The TRA posits that one's intention to perform or not perform a behavior is the "immediate determinant of that action" (Ajzen, 1985, p. 12). People are expected to act according to their intentions, excepting unanticipated situations. As intentions inevitably change, especially over longer periods of time, the accuracy of predictions is related to the time elapsed from measurement of the intention to observation of the behavior. The TRA further asserts that one's intention is a function of his positive or negative evaluation of performing the behavior, or attitude toward the behavior, and of his perception of the "social pressure" to perform or not perform the behavior, termed subjective norm (Ajzen, 1985, p. 12). A person typically intends to perform a behavior when they have a positive evaluation of the behavior and believe that other important figures expect them to perform the behavior.

The TRA's application is limited in that it fails to address behaviors under nonvolitional control and has low predictive validity when intentions are not current (Ajzen, 1985). As time elapses and new information becomes available, one's beliefs may change and may influence one's attitude toward the behavior or their subjective norm, thereby changing the intention. 
Invariably, every behavior is subject to nonvolitional influence (Ajzen, 1985). Broadly, one's volitional control over a behavior is related to how they perceive their own ability to control the behavior. More specifically, one must have the necessary information, skills, and abilities required to perform the behavior in question. Power of will, emotions, and compulsions also influence successful performance of an intended behavior.

External factors, namely time, opportunity, and dependence on other people, prevent performance of an intended behavior (Ajzen, 1985). These concepts differ from the circumstances that change a person's intentions. These external factors are nonvolitional barriers that force a change in behavior but do not change the person's attitude toward the behavior or the subjective norm.

The TPB is a modification of the TRA that considers perceived control over behavior in addition to actual control (Ajzen, 1985). It takes into account those unanticipated events that can disrupt the intention-behavior association. The TPB reframes the behavioral intention as the intention to attempt performing the behavior. The successful performance of the intended behavior depends on one's control over other influential factors that may impede their efforts. Intention is a more accurate predictor of attempted behavior than of actual performance. The strength of one's attempt to perform a behavior interacts with the degree of their control. The weight of each depends on the behavior. In line with the TRA, the intention is a function of attitude toward attempting to perform the behavior and of subjective norm about attempting. In this case, the attitude toward attempting is determined by the attitude toward successful performance and toward unsuccessful performance. Subjective probabilities of both succeeding and failing 
are also considered. These subjective probabilities are related to beliefs about internal and external factors that may influence performance. Behavioral expectation refers to a person's subjective probability that they will actually perform the behavior based on their intention to attempt and on the subjective probability that they can control it. The correlation between behavioral expectation and actual behavior depends on the relationship between one's personal belief in their control over the behavior and how much control they actually have, and thus, is reliant on an accurate assessment of these factors.

The TBP is a useful theory that provides guidance on assessing nurses' personal beliefs in the control over their own behavior regarding mobilizing patients. Assessing factors that influence nurses' perceptions in the domains of knowledge, attitude, and behaviors is the first step in determining the correlation between behavioral expectation and actual behavior. The methodology will be discussed next. 


\section{Methods}

\section{Design}

The design was a quantitative nonexperimental study consisting of a survey to identify nurses' perceived barriers to mobilizing patients. The key variables were perceived barriers in three domains: knowledge, attitudes, and behaviors.

\section{Site and Sample Selection}

The site for this project was a 40-bed medical-surgical unit of the communitybased Sturdy Memorial Hospital (SMH) in Attleboro, Massachusetts. The unit is adult inpatient and is the pilot Acute Care for Elders (ACE) unit of the hospital. The sample was a convenience sample of all the full-time, part-time, per-diem, and temporary agency nurses who predominantly worked on the targeted unit during the survey $(N=87)$.

\section{Procedures}

\section{Logic Model}

The Logic Model was used to guide this project. The connection between planned work and intended results is illustrated in the five-step diagram displayed in Figure 1: 


\section{Figure 1}

The Basic Logic Model

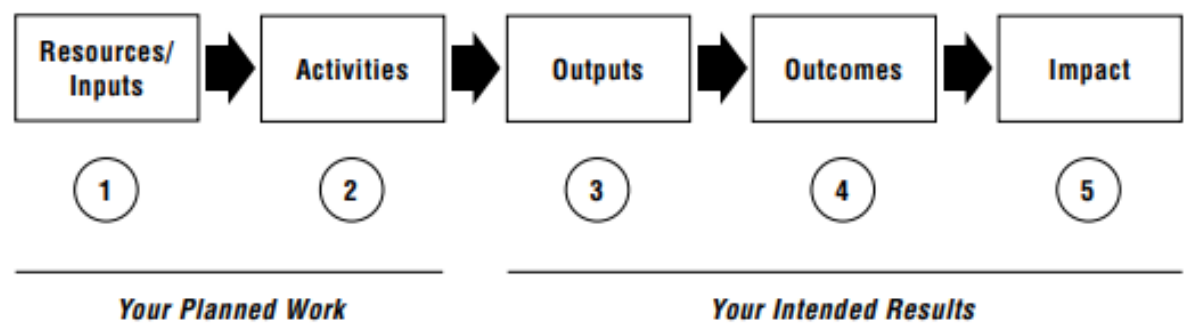

Note. From "Using Logic Models to Bring Together Planning, Evaluation, and Action: Logic Model Development Guide,” by W. K. Kellogg Foundation, 2004 (https://www.wkkf.org/resourcedirectory/resource/2006/02/wk-kellogg-foundation-logic-model-development-guide). Reprinted with the permission of the W. K. Kellogg Foundation.

The planned work included anticipated resources, or inputs, needed and intended actions or activities. Inputs are the human, financial, organizational, and community resources that will be needed and that are available to dedicate to the project. Activities are the processes, tools, events, technology, and actions that are implemented to achieve the intended outcomes. The intended results are all desired results of the project, including outputs, outcomes, and impact. Outputs are the direct products of the project activities. Outcomes are the changes in project participants' behavior, knowledge, skills, status, and level of functioning. Impact is the basic intended or unintended change that occurs in organizations, communities or systems as a result of project activities within 7-10 years (W. K. Kellogg Foundation, 2014). 


\section{Resources/Inputs}

This study proposal was submitted to the Director of Inpatient Nursing Services at $\mathrm{SMH}$, the unit manager of the targeted unit, and the SMH Institutional Review Board (IRB) for review and approval. It was then submitted to the Rhode Island College IRB for approval.

SMH leadership were supportive of educational advancement and welcomed a quality improvement project. They were also motivated to increase mobility of patients, especially older adult patients, having recently consulted with NICHE, nurse-driven program designed to help healthcare organizations improve care of older adults through nursing education (NICHE, 2020). A major NICHE initiative is to increase mobility to reduce functional decline and improve outcomes.

\section{Activities}

The Overall Provider Barriers scale is a validated self-administered survey developed by Hoyer et al. (2015) to assess the perceived barriers of direct patient care providers. The developers of this survey reported acceptable internal consistency reliability and construct validity. The survey was adapted for use in this study (Appendix A). Questions regarding provider characteristics (hospital site, discipline, and years of experience) were omitted. A convenience sampling method was used. All full-time, parttime, per-diem, and temporary agency nurses who predominantly worked on the targeted unit during survey distribution were eligible for inclusion. Float nurses and leadership staff were excluded. Recruitment for participation occurred via start of shift huddles and email 2 weeks prior to survey distribution. The survey was available in paper form distributed by huddle leaders and the unit educator, and an electronic survey was created 
using the online survey creator SurveyMonkey (www.surveymonkey.com) and sent out via email. The survey was available for 3 weeks. The project purpose was described to all eligible participants in posted fliers (Appendix B) and a letter (Appendix C), which also stated that participation was voluntary, participants could withdraw at any time, and that there would be no consequences for choosing not to participate in the project. The fliers and letter also included the principal investigator's contact information and explained participants' right to ask questions. The fliers were posted on the unit to increase awareness and notify staff of the study and of voluntary participation 2 weeks prior to the start of the survey.

The survey included instructions for the participants to provide responses on a 5point Likert scale to 26 statements, in three domains of knowledge, attitudes, and behaviors, that most accurately reflected their perceptions based on their experience in the $1-2$ weeks prior to taking the survey.

\section{Outputs}

Anonymous paper survey responses were collected in an envelope kept in the locked office of the unit educator. Electronic responses, without any identifying personal information, were collected through the SurveyMonkey website. Paper and electronic responses were pooled and transcribed into an Excel spreadsheet. Data was reviewed for completeness and patterns in responses. Descriptive analysis was used to evaluate the outcomes. 


\section{Outcomes}

The desired outcome was identification of the target population's perceived barriers to mobilizing patients. Mean rating scores of each survey item were calculated. Mean rating scores were compared to determine the highest perceived barrier. Mean rating scores of all survey items within each domain were calculated and compared to determine the domain with the most barriers. For each survey item, the percentage of respondents who indicated that the item was a barrier was calculated.

\section{Impact}

The survey responses were analyzed using descriptive analysis and presented in table form to depict the items perceived as barriers categorized by domain. The findings will be disseminated to SMH leadership staff to help guide future education of the targeted unit's nursing staff and aid in the development of a mobility protocol. Findings will also be presented at the RIC research symposium in May 2021. A barrier to data collection was low participation. Lack of a conceptual definition of mobility may have impacted responses. Next, the results will be discussed. 


\section{Results}

A total of 28 of the 87 eligible nurses completed the survey (response rate $=$ $32.18 \%$ ). The response rate was $3.45 \%$ for nurses completing the paper survey and $28.74 \%$ for nurses completing the electronic survey. All surveys were $100 \%$ completed. The perceived barriers to mobilizing patients were measured with a 5-point Likert scale that ranged from strongly disagree, disagree, neutral, agree, and strongly agree. Each barrier in the survey was in one of three domains. The survey items were not identified by domain on the survey, and items about barriers in knowledge, attitudes, and behaviors were mixed throughout the survey. The items alternated between negative and positive wordings. Negatively worded items were deemed indicative of a barrier if a participant had agreed or strongly agreed with the statement. Positively worded items were deemed indicative of a barrier if a participant had disagreed or strongly disagreed with the statement. For each item, the percentage of respondents who indicated that the item was a barrier was calculated. The mean rating score of each survey item was also calculated to allow for the inclusion of neutral responses in the data analysis. Positively worded items were reverse coded so that a higher mean rating score indicated a higher perception of a barrier. Responses were categorized into domains to identify the domain with the highest perceived barriers.

\section{Knowledge}

The mean percent of respondents who identified knowledge domain items as barriers was $8.93 \%$. The total mean score in the knowledge domain was 2.11 out of 5 . Respondents tended to agree with the knowledge domain statements, indicating that knowledge is not a highly perceived barrier. Based on a mean score of 2.36 , the highest 
perceived knowledge domain barrier was adequate training on how to safely mobilize patients $(14.29 \%$ of respondents disagreed or strongly disagreed with having received adequate training; item 2). While no respondents disagreed or strongly disagreed that they educate their patients to exercise or increase their physical activity while in the hospital, the mean score of 2.18 makes this the second highest perceived barrier in the knowledge domain (item 25). The highest perceived barrier as a percentage was understanding which inpatients are appropriate to refer to occupational therapy $(17.86 \%$ of respondents disagreed or strongly disagreed with the statement; $M=2.14$; item 6). Only $3.57 \%$ of respondents identified understanding which patients to refer to physical therapy as a barrier $(M=1.75$; item 5$)$.

\section{Attitudes}

The mean percent of respondents who identified attitudes domain items as barriers was $17.46 \%$. The total mean scores in the attitudes domain was 2.38 . The perception that increasing mobilization of patients will be more work for nurses was identified by $57.14 \%$ of respondents, making it the highest barrier in the attitudes domain and third highest overall ( $M=3.46$; item 12$)$. In contrast, $32.14 \%$ of respondents indicated that increasing mobilization of patients would be more work for physical and/or occupational therapist ( $M=2.75$; item 13$)$. The perception that a physical therapist or occupational therapist should be the primary care provider to mobilize patients was the second highest barrier, based on the mean score of 2.75 (14.29\% of respondents agreed or strongly agreed with the statement; item 4). Only $14.29 \%$ of respondents felt that their patients had time to be mobilized at least three times daily during their day $(M=2.54$; item 26). Even fewer respondents felt that their patients were too sick to be mobilized 
( $10.71 \%$ of respondents agreed or strongly disagree with the statement; $M=2.43$; item 1). Not feeling confident in their ability to mobilize their patients was identified by $17.86 \%$ of respondents $(M=2.29$; item 21$)$. Only $7.14 \%$ of respondents identified that they were not sure when it is safe to mobilize their patients $(M=2.04$; item 19). Of the 28 respondents, one respondent (3.57\%) strongly disagreed that inpatients who are mobilized at least three times daily will have better outcomes $(M=1.61$; item 18$)$. None of the respondents indicated that increasing mobilization of inpatients would be harmful to them $(M=1.54$; item 3$)$.

\section{Behaviors}

Items in the behaviors domain tended to have the highest percentages of perceived barriers and the highest mean scores. The mean percent of respondents who identified behaviors domain items as barriers was $29.40 \%$. The total mean score in the behaviors domain was 2.82. Overall, inadequate nurse-to-patient staffing was found to be the highest perceived barrier to mobilizing patients, identified by $75 \%$ of respondents $(M=$ 3.86; item 9). The second overall highest perceived barrier was time, with $60.71 \%$ of respondents agreeing or strongly disagreeing with having time to mobilize their patients during their shift/workday $(M=3.61$; item 23$)$. The third highest perceived barrier in the behaviors domain was that patients are resistant to being mobilized, identified by $35.71 \%$ of respondents $(M=3.25$; item 17$)$. The remainder of the perceived barriers are listed from highest to lowest based on the mean scores: infrequent family member interest in helping mobilize patients $(28.57 \%$ of respondents disagreed or strongly disagreed that family members are frequently interested; $M=3.04$; item 20); lack of regular discussion of patients' physical functioning between healthcare providers $(28.57 \%$ of respondents 
disagreed or strongly disagreed that physical functioning is regularly discussed; $M=$ 2.71 ; item 8$)$; mobilization of patients by nurses (32.14\% of respondents disagreed or strongly disagreed that nurses mobilized patients at least once daily; $M=2.71$; item 11); lack of appropriate physician orders to mobilize patients $(25 \%$ of respondents disagreed or strongly disagreed that patients usually have appropriate orders; $M=2.68$; item 16 ); lack of proper equipment and/or furnishings (28.57\% of respondents agreed or strongly agreed that they did not have the proper equipment and/or furnishings; $M=2.68$; item 7); lack of mobilization of the patient by the respondents (17.86\% disagreed or strongly disagreed that they mobilize their patients at least once during their shift/work day; $M=$ 2.68; item 24); patient contraindications to being mobilized ( $14.29 \%$ of respondents agreed or strongly disagreed that patients often have contraindications; $M=2.57$; item 10); documentation of the physical functioning of the patient by the respondent during their shift/work day $(14.29 \%$ of respondents disagreed or strongly disagreed; $M=2.46$; item 22); and the belief that increasing the frequency of mobilizing patients increases the respondent's risk for injury ( $14.29 \%$ of respondents agreed or disagreed; $M=2.43$; item 15). Only one respondent identified that departmental leadership is not very supportive of patient mobilization $(3.75 \% ; M=2.11$; item 14$)$. See Table 1 for the list of items from all domains and the percentage of respondents who indicated the items as barriers and not as barriers. 


\section{Table 1}

Percentage of Respondents Who Indicated Disagreement and Agreement with Individual Survey Items

Survey Items
Disagree

Strongly

disagree
Agree

Strongly agree

\section{Knowledge Domain}

2. I have received training on how to safely mobilize my inpatients.

$14.29 \%$

$67.86 \%$

5. I understand which inpatients are appropriate to refer to physical therapy.

$3.57 \%$

$85.71 \%$

6. I understand which inpatients are appropriate to refer to occupational therapy.

$17.86 \%$

$78.57 \%$

25. Unless there is a contraindication, I educate my inpatients to exercise or increase their physical activity while on my hospital unit.

0

$71.43 \%$

\section{Attitudes}

1. My inpatients are too sick to be mobilized.

$64.29 \%$

$10.71 \%$

3. Increasing mobilization of my inpatients will be harmful to them.

$96.43 \%$

0

4. A physical therapist or occupational therapist should be the primary care provider to mobilize my inpatients.

$42.86 \%$

$14.29 \%$

12. Increasing mobilization of my inpatients will be more work for nurses.

$35.71 \%$

$57.14 \%$

13. Increasing mobilization of my inpatients will be more work for physical and/or occupational therapists.

$60.71 \%$

$32.14 \%$

18. I believe that my inpatients who are mobilized at least three times daily will have better outcomes.

$3.57 \%$

$89.29 \%$

19. I am not sure when it is safe to mobilize my inpatient.

$78.57 \%$

$7.14 \%$

21. I do not feel confident in my ability to mobilize my inpatients.

$71.43 \%$

$17.86 \%$

26. My patients have time during their day to be mobilized at least three times daily.

$14.29 \%$

$57.14 \%$

\section{Behaviors}

7. We don't have the proper equipment and/or furnishings to mobilize my inpatients. 
8. The physical functioning of my inpatients is regularly discussed between the patient's healthcare providers (nurses, physicians, physical therapists, occupational therapists).

$28.57 \% \quad 46.43 \%$

9. Nurse-to-patient staffing is adequate to mobilize inpatients on my unit(s).

$75.00 \%$

$10.71 \%$

10. My inpatients often have contraindications to be mobilized.

$57.14 \%$

$14.29 \%$

11. Unless there is a contraindication, my inpatients are mobilized at least once daily by nurses.

$32.14 \%$

$46.43 \%$

14. My departmental leadership is very supportive of patient mobilization.

$3.57 \%$

$67.86 \%$

15. Increasing the frequency of mobilizing my inpatients increases my risk for injury.

$64.29 \%$

$14.29 \%$

16. Inpatients who can be mobilized usually have appropriate physician orders to do so.

17. My inpatients are resistant to being mobilized.

20. Family members of my inpatients are frequently interested to help mobilize them.

$28.57 \%$

$25.00 \%$

22. I document the physical functioning status of my inpatient during my shift/workday.

\section{I do not have time to mobilize my inpatients during} my shift/workday.

24. Unless there is a contraindication, I mobilize my inpatients at least once during my shift/workday.

Note. The four items identified as barriers by the greatest percentage of respondents are bolded.

The summary and conclusions will be discussed next. 


\section{Summary and Conclusions}

Previous studies have found no benefit from interventions aimed at increasing nurses' knowledge of the importance of mobilizing patients. In one quasi-experimental study on the implementation of a nurse-driven mobility protocol, ambulation did not improve after nurses and patient care technicians were educated through verbal presentations and self-learning packets (Drolet et al., 2013). In another study, the perception of increased workload for nurses was found to be the highest barrier (Hoyer at al., 2015). Nurses have been observed to infrequently mobilize ambulatory patients who require human assistance (Doherty-King et al., 2014). In the study by Doherty-King et al. (2014), all dependent patients' ambulation events were initiated by the patient. In a study that evaluated the impact of MOVE interventions, $51 \%$ of patients reported that education activities and materials were effective in increasing their knowledge about the importance of mobilizing during their hospitalization, and $40 \%$ of patients reported that educational activities were effective in helping them mobilize (Holroyd-Leduc et al., 2019).

In this project, lack of knowledge related to mobilizing patients was not found to be a high barrier. Interventions targeted at other domains, specifically behaviors, may have more impact. Barriers in the behaviors domain were perceived as the greatest barriers, with inadequate staffing and lack of time as the two highest barriers and patient resistance to mobilization as the fourth highest perceived barriers. The perception of increased workload for nurses (attitudes) was found to be the third highest barrier in this project. Given that the respondents perceived that patient mobilization would increase nurses' workloads, it is not surprising that lack of time and adequate staffing were also 
perceived as barriers. This quality improvement project at SMH found that $46.43 \%$ of respondents agreed or strongly agreed that their patients are mobilized at least once daily by nurses; $32.14 \%$ of respondents disagreed or strongly disagreed (raw $M=3.29$; item 11; behaviors). Half of the respondents agreed or strongly agreed with the statement that they personally mobilized their patients at least once during their shift, while $17.86 \%$ disagreed or strongly disagreed and $32.14 \%$ were neutral (raw $M=3.32$; item 24; behaviors). The survey did not differentiate mobility events. It is plausible that the low mobilization reported by the respondents was related to lack of time and or inadequate staffing.

The third highest barrier identified by $35 \%$ of the respondents of this survey was patient resistance to mobilization (behaviors). Patient education was not identified as a perceived barrier (knowledge) in this project, but the survey did not collect information on the quality or effectiveness of the education. Standardization of patient education about mobilization may improve the quality and effectiveness of the education, which may decrease patient resistance to mobilization.

This quality improvement project aimed at identifying nurses' perceived barriers to mobilizing patients on a medical-surgical unit is the first step toward creating barrier targeted interventions aimed at increasing nurse engagement in mobilizing patients on the targeted unit. Increasing engagement of the nurses is an identified goal of SMH leadership staff in accordance with the NICHE vision of improving the care of hospitalized older adults. 


\section{Limitations}

During the final week of survey availability, patient visitation guidelines changed. For the first 15 days that the survey was available, visitors were not allowed in the hospital, except for special circumstances, due to the ongoing COVID-19 pandemic. During days 16-21, limited visitation was allowed. This may have impacted responses regarding family members' interest in helping mobilize patients.

The response rate remained low, despite survey availability electronically and on paper. Given that respondents identified lack of time to mobilize their patients as a barrier, lack of time was perhaps a barrier to completing a survey during their shift. A QR code posted in the staff breakroom and or a survey link sent through the hospital's textmessaging system may have made survey access easier and increased the response rate.

Next, recommendations and implications for advanced nursing practice will be discussed. 


\section{Recommendations and Implications for Advanced Nursing Practice}

Leaders at SMH have demonstrated a commitment to improving care for hospitalized elders by partnering with NICHE. One of the major NICHE strategies to improve the outcomes of hospitalized elders is promoting mobilization. To promote mobilization to improve outcomes, SMH leaders identified the need to increase nurse engagement in mobilizing their older adult population. With only half of the nurses responding in this survey that they agreed that they personally mobilize their patients at least once during their shift, the results suggest that patient mobilization is not a high priority. Determining the reasons why nurses do not mobilize patients is essential to creating a sustainable plan for change. The information obtained from the surveys about nurses' perceived barriers will be used to address those barriers and create targeted interventions to increase nurse engagement in patient mobilization.

Advanced practice registered nurse, clinical nurse specialists (APRN-CNSs) are change agents with expertise in problem identification, gap analysis, and process and outcomes measurement, ideally suited to lead evidence-based practice change to improve patient outcomes. The information provided by this quality improvement project will be used to develop interventions targeted at the highest barriers to patient mobilization. To address time and staffing barriers, the APRN-CNS will work collaboratively and creatively with leadership and unit staff to determine ways to minimize inefficiencies and maximize time and resources, including human resources. Nurses and nurse aides will be engaged in focus groups to discuss the barriers and potential solutions. Additional information may be gathered in future survey and quality improvement projects to identify collaborative efforts to improve mobilization with other members of unit staff. 
The APRN-CNS is uniquely prepared to provide leadership of a unit-based team to address patient resistance to mobilization as a barrier, evaluate current patient mobility education, and develop an evidence-based practice project to increase patient knowledge of the benefits of mobilization and participation in mobility activities. Standardized, evidence-based education may increase patient knowledge and willingness to mobilize.

The APRN-CNS is uniquely positioned to develop a team of mobility champions to develop an evidence-based mobility protocol based on the unit's strengths and barriers that were identified through the quality improvement projects. The protocol will include evidence-based patient education and patient engagement. The unit's leaders, who are interested in creating and supporting an Acute Care for Elders (ACE) unit, will partner with the APRN-CNS and the team to plan and implement the quality improvement projects and mobility protocol on the targeted unit. Additional stakeholders, including patients, staff nurses, nurse aides, physical therapists, occupational therapists, respiratory therapists, and dietitians will be recruited to form a multidisciplinary team to assist with the process. The APRN-CNS will disseminate the results of the changes that were successfully piloted on the targeted unit and assist leaders and unit staff of the other SMH inpatient units in adopting or adapting the changes.

Previous research discussed in this paper has demonstrated that ambulation improved after daily reports with patient's LOS and amount of ambulation on a given day were given to the nurses (Drolet et al., 2013). Clear expectations for ambulation at the unit level had the most significant impact on whether nurses ambulated patients (Doherty-King \& Bowers, 2011). This is consistent with the TRA assertion that a person typically intends to perform a behavior when they have a positive evaluation of the 
behavior and believe that other important figures expect them to perform the behavior. This survey did not include items regarding mobility expectations. Only one respondent in this project disagreed that their departmental leadership was supportive of patient mobilization (behaviors). The results of this project and the previous research findings may support regular communication between leadership and unit staff. The mobility protocol will improve the clarity of expectations for mobility through standardized order sets. Leadership, in partnership with the multidisciplinary quality improvement team members, will regularly discuss progress toward outcome goals.

The literature surrounding mobility of hospitalized patients comes from low-level evidence, largely. Future research should focus on studies that examine outcomes related to hospitalized patient mobility levels and mobility protocols and initiatives. The APRNCNS is ideally suited to lead research studies aimed at addressing this gap in the research. 


\section{References}

Ajzen, I. (1985). From intentions to actions: A theory of planned behavior. In J. Kuhl \& J. Beckman (Eds.), Action-control: From cognition to behavior (pp. 11-39). Heidelberg, Germany: Springer. https://doi.org/10.1007/978-3-642-69746-3_2

Brown, C. J., Redden, D. T., Flood, K. L., \& Allman, R. M. (2009). The underrecognized epidemic of low mobility during hospitalization of older adults. Journal of the American Geriatrics Society, 57(9), 1660-1665. doi: 10.1111/j.15325415.2009.02393.x

Castro, E., Turcinovic, M., Platz, J., \& Law, I. (2015). Early mobilization: Changing the mindset. Critical Care Nurse, 35(4), 1-7. http://dx.doi.org/10.4037/ccn2015512

Coker, R.H., Hays, N.P., Williams, R.H., Wolfe, R.R., \& Evans, W.J. (2015). Bed rest promotes reductions in walking speed, functional parameters, and aerobic fitness in older, healthy adults. Journals of Gerontology, Series A: Biological Sciences and Medical Sciences, 70(1), 91-96. doi:10.1093/gerona/glu123

Cortez, R. (2018). Geriatric trauma protocol. Journal of Trauma Nursing, 25(4), 218-227. doi: 10.1097/JTN.000000000000037

Dirks, M. L., Wall, B. T., van de Valk, B., Holloway, T. M., Holloway, G. P., Chabowski, A., Goossens, G. H., \& van Loon, L. J. C. (2016). One week of bed rest leads to substantial muscle atrophy and induces whole-body insulin resistance in the absence of skeletal muscle lipid accumulation. Diabetes, 65(10), 28622875. https://doi.org/10.2337/db15-1661 
Doherty-King, B., \& Bowers, B. (2011). How nurses decide to ambulate hospitalized older adults: Development of a conceptual model. The Gerontologist, 51(6), 786797. doi:10.1093/geront/gnr044

Doherty-King, B., Yoon, J.Y., Pecanac, K., Brown, R., \& Mahoney, J. (2014). Frequency and duration of nursing care related to older patient mobility. Journal of Nursing Scholarship, 46(1), 20-27. doi: 10.1111/jnu.12047

Drolet, A., DeJuilio, P., Harkless, S., Henricks, S., Kamin, E., Leddy, E.A., Lloyd, J.M., Waters, C., \& Williams, S. (2013). Move to improve: The feasibility of using an early mobility protocol to increase ambulation in the intensive and intermediate care settings. Physical Therapy, 93(2), 197-207. https://doi.org/10.2522/ptj.20110400

Drummond, M., Dickinson, J.M., Fry, C.S., Walker, D.K., Gundermann, D.M., Reidy, P.T., Timmerman, K.L., Markofski, M.M., Paddon-Jones, D., Rasmussen, B.B., \& Volpil, E. (2012). Bed rest impairs skeletal muscle amino acid transporter expression, mTORC1 signaling, and protein synthesis in response to essential amino acids in older adults. American Journal of Physiology - Regulatory, Integrative and Comparative Physiology, 302, 1113-1122. Doi:

10.1152/ajpendo.00603.2011.

Drummond, M.J., Timmerman, K.L., Markofski, M.M., Walker, D.K., Dickinson, J.M., Dickinson, M., Brasier, A.R., Rasmussen, B.B., \& Volpil, E. (2013). Short-term bed rest increases TLR4 and IL-6 expression in skeletal muscle of older adults. American Journal of Physiology - Regulatory, Integrative and Comparative Physiology, 305, R216-R223. doi:10.1152/ajpregu.00072.2013 
Fisher, S. R., Goodwin, J. S., Protas, E. J., Kuo, Y-K., Graham, J. E., Ottenbacher, K. J., \& Ostir, G. V. (2011). Ambulatory activity of older adults hospitalized with acute medical illness. Journal of the American Geriatrics Society, 59, 91-95. doi: 10.1111/j.1532-5415.2010.03202.x

Hoyer, E. H., Brotman, D. J., Chan, K., \& Needham, D. M. (2015). Barriers to early mobility of hospitalized general medicine patients: Survey development and results. American Journal of Physical Medicine and Rehabilitation, 94(4), 304312. doi:10.1097/PHM.0000000000000185

Jones, R.J., Merkle, S., Ruvalcaba, L., Ashton, P., Bailey, C., \& Lopez, M. (2019). Nurse-led mobility program: Driving a culture of early mobilization in medicalsurgical nursing. Journal of Nursing Care Quality 35(1), 20-26. doi:

10.1097/NCQ.0000000000000404

King, B. J., Steege, L. M., Winsor, K., VanDenbergh, S., \& Brown, C. J. (2016). Getting patients walking: A pilot study of mobilizing older adult patients via a nursedriven intervention. Journal of the American Geriatrics Society, 64, 2088-2094. doi: $10.1111 /$ jgs. 14364

Liu, B., Moore, J.A., Almaawiy, U., Chan, W., Khan, S., Ewusie, J., Hamid, J.S., \& Straus, S.E. (2017). Outcomes of Mobilisation of Vulnerable Elders in Ontario (MOVE ON): a multisite interrupted times series evaluation of an implementation intervention to increase patient mobilisation. Age and Ageing, 47, 112-119. doi: 10.1093/ageing/afx128 
Mudge, A. M., McRae, P., McHugh, K., Griffin, L., Hitchen, A., Walker, J., Cruickshank M., Morris, N. R., Kuys, S. (2016). Poor mobility in hospitalized adults of all ages. Journal of Hospital Medicine, 11(4) 289-291. doi:10.1002/jhm.2536

Nurses Improving Care for Healthsystem Elders. (2020, February 6). Our Story. NICHE. https://nicheprogram.org/

Padula, C. A., Hughes, C., \& Baumhover, L. (2009). Impact of a nurse-driven mobility protocol on functional decline in hospitalized older adults. Journal of Nursing Care Quality, 24(4), 325-331. doi: 10.1097/NCQ.0b013e3181a4f79b

Pedersen, M.M., Bodilsen, A.C., Petersen, J., Beyer, N., Andersen, O., Lawson-Smith, L., Kehlet, H., \& Bandholm, T. (3013). Twenty-four hour mobility during acute hospitalization in older medical patients. Journals of Gerontology, Series A: Biological Sciences and Medical Sciences, 68(3), 331-337. doi:10.1093/gerona/gls165

Qu, H., Li, Z., Zhai, Z., Liu, C., Wang, S., Guo, S., \& Zhang, Z. (2015). Predicting of venous thromboembolism for patients undergoing gynecological surgery. Medicine, 94(39), 1653-1658. doi: 10.1097/MD.0000000000001653

Ringholm, S., Bienso, R.S., Kiilerich, K., Guadelupe-Grau, A., Aachmann-Andersen, N.J., Saltin, B., Plomgaard, P., Lundby, C., Wojtaszewski, J.F.P., Calbet, J.A., \& Pilegaard, H. (2011). Bed rest reduces metabolic protein content and abolishes exercise-induced mRNA responses in human skeletal muscle. American Journal of Physiology-Endocrinology and Metabolism, 301, 649-658.

doi:10.1152/ajpendo.00230.2011 
Silke, T., Drabik, A., \& Stock, S. (2011). Pressure ulcers in older hospitalised patients and its impact on length of stay: a retrospective observational study. Journal of Clinical Nursing, 21, 380-387. https://doi.org/10.1111/j.1365-2702.2011.03915.x

W. K. Kellogg Foundation. (2004). Using logic models to bring together planning, evaluation, and action: Logic Model development guide. W. K. Kellogg Foundation. https://www.wkkf.org/resource-directory/resource/2006/02/wkkellogg-foundation-logic-model-development-guide

Zisberg, A., Agmon, M., Gur-Yaish, N., Rand, D., Hayat, Y., \& Gil, E. (2018). No one size fits all—the development of a theory-driven intervention to increase inhospital mobility: the "WALK-FOR" study. BMC Geriatrics 18(91). https://doi.org/10.1186/s12877-018-0778-3

Zisberg, A., Shadmi E., Sinoff, G., Gur-Yaish, N., Srulovici, E., \& Admi, H. (2011). Low mobility during hospitalization and functional decline in older adults. Journal of American Geriatric Society, 59(2), 266-73. https://doiorg.ric.idm.oclc.org/10.1111/j.1532-5415.2010.03276.x 


\section{Appendix A}

\section{Modified Overall Provider Barriers Scale}

Please circle a response that most accurately reflects your opinions based on your experience during the past 1-2 weeks.

\begin{tabular}{|c|c|c|c|c|c|}
\hline & $\begin{array}{l}\text { Strongly } \\
\text { disagree }\end{array}$ & Disagree & Neutral & Agree & $\begin{array}{c}\text { Strongly } \\
\text { agree }\end{array}$ \\
\hline 1. My inpatients are too sick to be mobilized. & 1 & 2 & 3 & 4 & 5 \\
\hline $\begin{array}{l}\text { 2. I have received training on how to safely } \\
\text { mobilize my inpatients. }\end{array}$ & 1 & 2 & 3 & 4 & 5 \\
\hline $\begin{array}{l}\text { 3. Increasing mobilization of my inpatients will } \\
\text { be harmful to them. }\end{array}$ & 1 & 2 & 3 & 4 & 5 \\
\hline $\begin{array}{l}\text { 4. A physical therapist or occupational } \\
\text { therapist should be the primary care provider to } \\
\text { mobilize my inpatients. }\end{array}$ & 1 & 2 & 3 & 4 & 5 \\
\hline $\begin{array}{l}\text { 5. I understand which inpatients are } \\
\text { appropriate to refer to physical therapy. }\end{array}$ & 1 & 2 & 3 & 4 & 5 \\
\hline $\begin{array}{l}\text { 6. I understand which inpatients are } \\
\text { appropriate to refer to occupational therapy. }\end{array}$ & 1 & 2 & 3 & 4 & 5 \\
\hline $\begin{array}{l}\text { 7. We don't have the proper equipment and/or } \\
\text { furnishings to mobilize my inpatients. }\end{array}$ & 1 & 2 & 3 & 4 & 5 \\
\hline $\begin{array}{l}\text { 8. The physical functioning of my inpatients is } \\
\text { regularly discussed between the patient's } \\
\text { healthcare providers (nurses, physicians, } \\
\text { physical therapists, occupational therapists). }\end{array}$ & 1 & 2 & 3 & 4 & 5 \\
\hline $\begin{array}{l}\text { 9. Nurse-to-patient staffing is adequate to } \\
\text { mobilize inpatients on my unit(s). }\end{array}$ & 1 & 2 & 3 & 4 & 5 \\
\hline $\begin{array}{l}\text { 10. My inpatients often have contraindications } \\
\text { to be mobilized. }\end{array}$ & 1 & 2 & 3 & 4 & 5 \\
\hline $\begin{array}{l}\text { 11. Unless there is a contraindication, my } \\
\text { inpatients are mobilized at least once daily by } \\
\text { nurses. }\end{array}$ & 1 & 2 & 3 & 4 & 5 \\
\hline $\begin{array}{l}\text { 12. Increasing mobilization of my inpatients } \\
\text { will be more work for nurses. }\end{array}$ & 1 & 2 & 3 & 4 & 5 \\
\hline
\end{tabular}


13. Increasing mobilization of my inpatients will be more work for physical and/or 1 2 3

4 5 occupational therapists.

14. My departmental leadership is very supportive of patient mobilization.

$\begin{array}{lllll}1 & 2 & 3 & 4 & 5\end{array}$

15. Increasing the frequency of mobilizing my inpatients increases my risk for injury.

12

23

34

5

16. Inpatients who can be mobilized usually have appropriate physician orders to do so.

$\begin{array}{lllll}1 & 2 & 3 & 4 & 5\end{array}$

17. My inpatients are resistant to being mobilized.

1

1

18. I believe that my inpatients who are mobilized at least three times daily will have 12

2

3

4 5 better outcomes.

\section{I am not sure when it is safe to mobilize my} inpatient.

12

23

3

4

5

20. Family members of my inpatients are frequently interested to help mobilize them.

$\begin{array}{lllll}1 & 2 & 3 & 4 & 5\end{array}$

21. I do not feel confident in my ability to mobilize my inpatients.

1

2

3

4

5

22. I document the physical functioning status of my inpatient during my shift/work day.

1

2

3

$4 \quad 5$

23. I do not have time to mobilize my inpatients during my shift/work day.

1

2

3

24. Unless there is a contraindication, I mobilize my inpatients at least once during my 1

2

3

$4 \quad 5$
shift/work day.

25. Unless there is a contraindication, I educate my inpatients to exercise or increase their 1 physical activity while on my hospital unit.

26. My patients have time during their day to be mobilized at least three times daily.

1

2

345

$\begin{array}{llll}2 & 3 & 4\end{array}$




\section{Appendix B}

\section{Recruitment Flier}

\section{Mont Nurses Needed}

I am looking for registered nurses to participate in a quality improvement project

This project consists of a 5-minute, 26-item survey

You will be asked to rate your agreement with statements about mobilizing patients

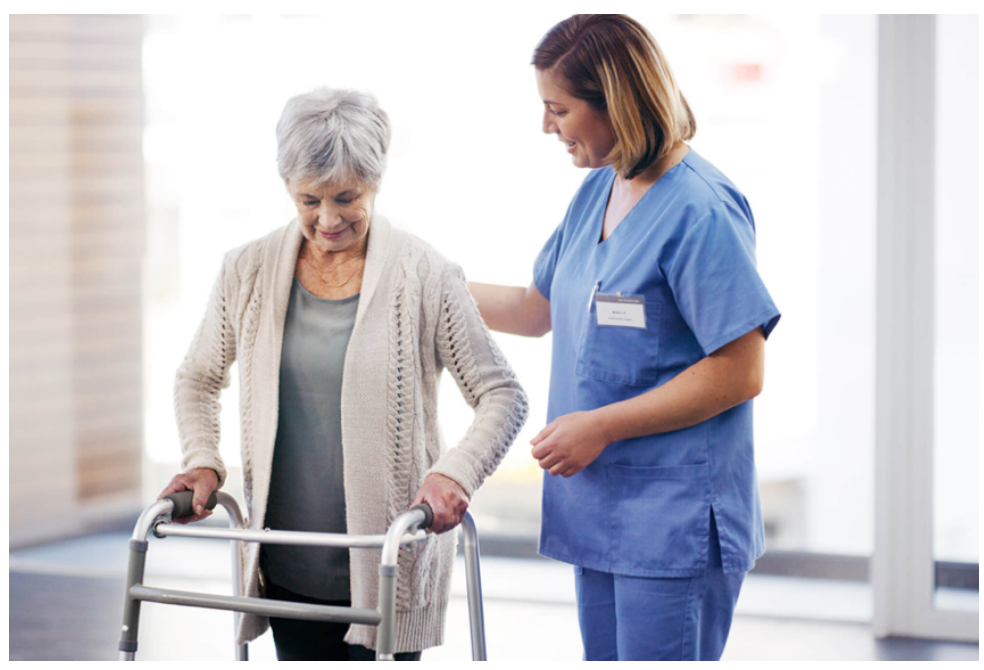

Who: Montplaisir registered nurses

What: Identifying Nurses' Perceived Barriers to Mobilizing Patients on a Medical-Surgical Unit: A Quality Improvement Project

Where: Obtain your survey at Huddle or through your work email

When: $2 / 14 / 21-3 / 6 / 21$

Contact: (508) 837-3518 or jlukin_5555@email.ric.edu

Kathleen Murphy Beksha (Principal Investigator)

Rhode Island College 
Appendix C

Recruitment Letter

Informational Letter

\section{Identifying Nurses' Perceived Barriers to Mobilizing Patients on a Medical-Surgical Unit: A Quality Improvement Project}

Montplaisir Nurses,

Jill Lukin, a clinical nurse specialist student in the Master of Science in Nursing degree program at Rhode Island College, along with the principal investigator, Kathleen Murphy Beksha, is completing a quality improvement project on Montplaisir as part of her Master's program. The purpose of this quality improvement project is to identify nurses' perceived barriers to mobilizing patients on a medical-surgical unit. To be eligible to participate, you must be a full-time, part-time, per-diem, or temporary agency nurse who predominantly works on Montplaisir. In order to complete this quality improvement project, you are being asked to participate. Participation is voluntary, and if you agree to participate, it will involve the completion of an anonymous survey. It will take approximately five minutes to complete this survey.

The survey will consist of 26 statements aimed at your attitudes, beliefs, and behaviors regarding mobilizing patients. Participation in the survey is voluntary, you may withdraw at any time, and there will be no consequences for choosing not to participate in the survey. No personal information will be collected.

If you have any questions or concerns regarding the project, you may contact me at (508) 837-3518 or jlukin_5555@email.ric.edu. You may also contact Professor Patricia Calvert, the Rhode Island College, School of Nursing advisor for this major project, at (401) 456-6323 or pcalvert@ric.edu or Kathleen Murphy Beksha at (617) 899-9509 or kbeksha@sturdymemorial.org. I look forward to working with the Montplaisir nurses and hope you will consider participation in this quality improvement project.

Thank you,

Jill Lukin 